\title{
ELF magnetic fluctuations detected by Kaguya in deepest lunar wake associated with type-II protons
}

\author{
Tomoko Nakagawa ${ }^{1 *}$, Tatsuya Nakashima ${ }^{1,2}$, Takuya Wada1,3, Hideo Tsunakawa ${ }^{4}$, Futoshi Takahashi5, \\ Hidetoshi Shibuya ${ }^{6}$, Hisayoshi Shimizu$^{7}$, Masaki Matsushima ${ }^{4}$ and Yoshifumi Saito ${ }^{8}$
}

\begin{abstract}
Magnetic fluctuations in the extremely low-frequency (ELF) range from 0.1 to $10 \mathrm{~Hz}$ were found by the Lunar Magnetometer (LMAG) of the magnetic field and plasma experiment (MAP) on board the spacecraft Kaguya in the deepest wake behind the moon, where the magnetic field is usually quiet. The fluctuations were compressional and non-monochromatic, showing no preferred polarization. They were often accompanied by "type-ll entry" solar wind protons that were reflected by the dayside lunar surface or crustal magnetic field, gyrated around the solar wind magnetic field, then entered the deepest wake. The ELF waves persisted for $30 \mathrm{~s}$ to several minutes. The duration was often shorter than that of the type-Il protons. Most of the waves were detected on the magnetic field lines disconnected from the lunar surface, along which the solar wind electrons were injected into the wake. Since a large cross-field velocity difference is expected between the type-ll protons and the solar wind electrons injected along the magnetic field, some cross-field current-driven instability such as the lower hybrid two-stream instability is expected to be responsible for the generation of the waves.
\end{abstract}

Keywords: Moon; Wake; Solar wind; Reflected protons; Magnetic field; ELF; Modified two-stream instability; Kaguya; Electron injection; Type-II entry

\section{Introduction}

Since the moon does not have a global magnetic field, solar wind particles hit the moon directly, and most of them are absorbed by the surface of the moon, leaving a plasma cavity called the lunar wake on the side of the moon that is downstream of the solar wind (Bosqued et al. 1996; Colburn et al. 1967; Lyon et al. 1967; Ness et al. 1968; Ogilvie et al. 1996; Owen et al. 1996; Schubert and Lichtenstein 1974).

Recent observations made by the spacecraft Kaguya revealed that not all solar wind particles are absorbed, but $0.1 \%$ to $1 \%$ of them are reflected by the lunar surface, the crustal magnetic field, or the anti-moonward electric field over the magnetic anomaly (Saito et al. 2008b, 2010, 2012). The reflected protons injected into the solar

\footnotetext{
*Correspondence: nakagawa@tohtech.ac.jp

${ }^{1}$ Tohoku Institute of Technology, 35-1 Yagiyama Kasumi-cho, Taihaku-ku, 982-8577, Sendai, Japan

Full list of author information is available at the end of the article
}

wind flow generate a variety of wave activity around the moon. Large amplitude, nearly monochromatic ultra-lowfrequency (ULF) waves of $0.01 \mathrm{~Hz}$ (Nakagawa et al. 2012; Tsunakawa et al. 2010) and non-monochromatic whistler waves within the extremely low-frequency (ELF) range from 0.03 to $10 \mathrm{~Hz}$ (Nakagawa et al. 2011; Tsugawa et al. 2012) were most commonly observed around the moon. Significant monochromatic whistler waves were also seen, although they were less common (Nakagawa et al. 2003; Tsugawa et al. 2011). The predominance of the two frequency bands is analogous to the low-frequency waves upstream of the Earth's bow shock, where the solar wind protons are reflected. The monochromatic, circularly polarized ULF waves are thought to be generated through the cyclotron resonance of the magnetohydrodynamic waves with the solar wind protons reflected by the moon, then convected down to be detected in the vicinity of the moon (Nakagawa et al. 2012). The generation of non-monochromatic whistler waves in the ELF range can

\section{Springer}

(c) 2015 Nakagawa et al: licensee Springer. This is an Open Access article distributed under the terms of the Creative Commons Attribution License (http://creativecommons.org/licenses/by/4.0), which permits unrestricted use, distribution, and reproduction in any medium, provided the original work is properly credited. 
be explained by the cyclotron resonance (Nakagawa et al. 2011; Tsugawa et al. 2012), although the mechanism has not been fully understood. Since the main source of these waves is the energy of the solar wind particles reflected at the dayside surface or over crustal magnetic fields of the moon, the wave activity is almost always observed on the dayside of the moon when it is exposed to the solar wind, and is depressed on the nightside. Usually, the center of the wake is magnetically quiet because of the absence of solar wind particles.




In spite of this, Kaguya sometimes, unexpectedly, detected magnetic fluctuations in the frequency range from 0.1 to $10 \mathrm{~Hz}$ in the center of the wake near the moon, in association with the solar wind protons that were once reflected at the dayside surface of the moon, were picked up by the convection electric field, then entered the center of the near wake because of their large Larmor radius, and are referred to as type-II entry ions (Nishino et al. 2009, 2010, 2013). The aims of this paper are to present the properties of the waves observed in the center of the wake and to discuss their generation mechanism.

\section{Instrumentation}

The magnetic field data used in this study were obtained by the lunar Lagnetometer (LMAG) of the magnetic field and plasma experiment (MAP) on board Kaguya (Shimizu et al. 2008; Takahashi et al. 2009; Tsunakawa et al. 2010) on its orbit encircling the moon at an altitude of $100 \mathrm{~km}$ during the period from 1 January 2008 to 30 September 2008. The period of the orbital motion was about $118 \mathrm{~min}$ at that altitude. The magnetic field vectors were obtained using a triaxial ring-core fluxgate sensor with a sampling frequency of $32 \mathrm{~Hz}$. The peak-to-peak noise was less than $0.1 \mathrm{nT}$ (Tsunakawa et al. 2010).

The ions and electrons were observed by the plasma energy angle and composition experiment (PACE) of MAP. The PACE instrument was composed of ion mass analyzer (IMA) and ion energy analyzer (IEA) for measuring ions below $28 \mathrm{keV} / \mathrm{q}$, together with electron spectrum analyzer (ESA)-S1 and ESA-S2 for electrons below $15 \mathrm{keV}$ (Saito et al. 2008a, 2008b). The IMA and ESA-S1 were installed on the spacecraft panel facing the lunar surface, while the IEA and ESA-S2 were installed on the opposite side (Saito et al. 2008a, 2010).

\section{ELF waves in central wake}

Figure 1a shows the dynamic spectrum of the ELF waves detected in the central wake on 24 September 2008. On this day, the moon was in the solar wind, and the orbital plane of Kaguya was close to the noon-midnight meridional plane (Figure 2). An enhancement of magnetic fluctuations in the ELF range up to $8 \mathrm{~Hz}$ was observed during a short period, from 10:10 to 10:12 in the center of the wake, where the magnetic field is usually quiet. The solar zenith angle was $150^{\circ}$ to $167^{\circ}$. The ELF wave was detected when the spacecraft was magnetically disconnected from the lunar surface. The red (blue) bars in Figure $1 \mathrm{~b}$ indicate the magnetic connection of the spacecraft to the dayside (nightside) surface of the moon, estimated from a simple extrapolation of the line of force of the magnetic field observed at Kaguya.

The magnetic fluctuation between 10:10 and 10:12 was accompanied by type-II protons, which are the solar wind protons that were once reflected by the dayside surface of the moon, were picked up by the solar wind electric field and entered the deepest wake (Nishino et al. 2009). Figure 1f shows the energy-time spectrum of ions measured by MAP-PACE/IMA. The type-II protons appeared at $10: 08$ around $0.3 \mathrm{keV}$ and persisted for more than 50 min. Electrons that are thought to be pulled into the wake because of the electric potential produced by the ions (Nishino et al. 2010) are also recognized in Figure 1c,d.

Figure 3a shows an example waveform of the ELF fluctuations observed during the period from 10:11:00 to 10:11:04, compared with a quiet period from 10:00:00 to 10:00:04 (Figure 3b) as a measure of the noise level. The waveform of the ELF wave was not an impulse or a step-like function. The largest amplitude of up to $0.4 \mathrm{nT}$ peak-to-peak was observed in the $B_{y \text {-sat }}$ component. The bottom panel of Figure 3a shows that there was a significant variation in the magnitude of the magnetic field $|B|$, suggesting that the magnetic variation was compressional.

Figure 4 compares the spectra of the magnetic field variations (a) during the period of ELF wave from 10:11:00 to $10: 11: 32$ and (b) during a quiet period from 10:00:00 to 10:00:32. In Figure 4a, the power density is enhanced in the frequency range from 0.3 to $10 \mathrm{~Hz}$ compared

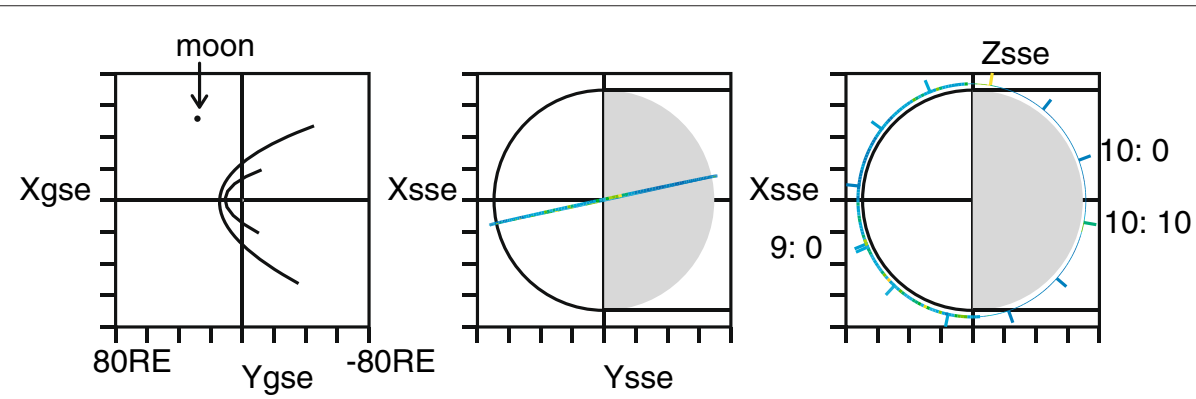

Figure 2 Orbit of Kaguya on 24 September 2008. (Left) in GSE coordinate system with respect to the Earth and the nominal bow shock, (center) northern view, and (right) meridional view from the duskside in SSE coordinates. The color of the curves indicates intensity of the magnetic fluctuations. 



Figure 3 Examples of waveforms of magnetic field variations. During (a) the magnetically fluctuating period from 10:11:00 to 10:11:04 and (b) a quiet period from 10:00:00 to 10:00:04, both on 24 September 2008, presented in a coordinate system fixed to the satellite. An artificial noise pattern that repeated every $1 \mathrm{~s}$ was removed from the original signals in advance. The artificial noise pattern was derived by a box-car method applied to signals of each component for an extremely quiet period, 10:00 to 10:05.

with a quiet period shown in Figure 4b. No distinct peak frequency was observed.

An attempt was made to obtain the direction of the wave number vector using minimum variance analysis, but the ratio of the intermediate to minimum variance was not large enough to obtain the minimum variance direction with a good accuracy. No clear polarization was found either.
Figure 5 shows another example of the magnetic fluctuations in the ELF range detected in the center of the wake between 00:08 and 00:12 on 29 September 2008. Again, the ELF waves were accompanied by type-II protons. It should be noted that the ELF waves were detected when the spacecraft was magnetically disconnected from the lunar surface. The electron flux was enhanced at the same time. 


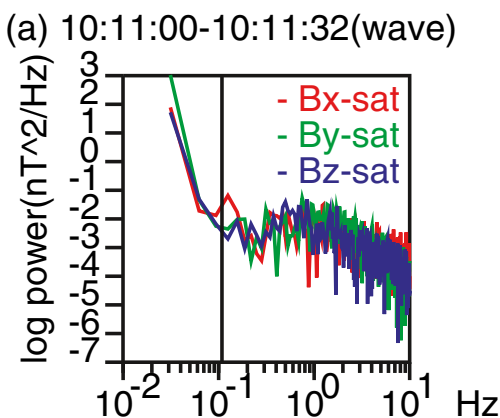

(b) 10:00:00-10:00:32(quiet)

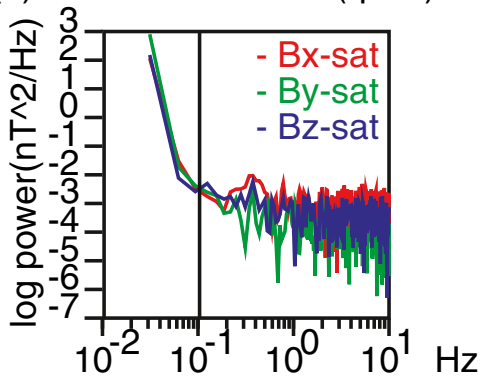

Figure 4 Spectra of magnetic fluctuations. During (a) the magnetically fluctuating period from 10:11:00 to 10:1 1:32 on 24 September 2008 and (b) a quiet period from 10:00:00 to 10:00:32 on 24 September 2008. Red, green, and blue curves are for $B_{x-\text { sat, }} B_{y-\text { sat, }}$ and $B_{z-\text { sat }}$ components in spacecraft coordinates, respectively. Vertical lines indicate proton cyclotron frequencies. Noise reduction as employed in Figure 3 and the Hamming window were applied prior to the Fourier transform.

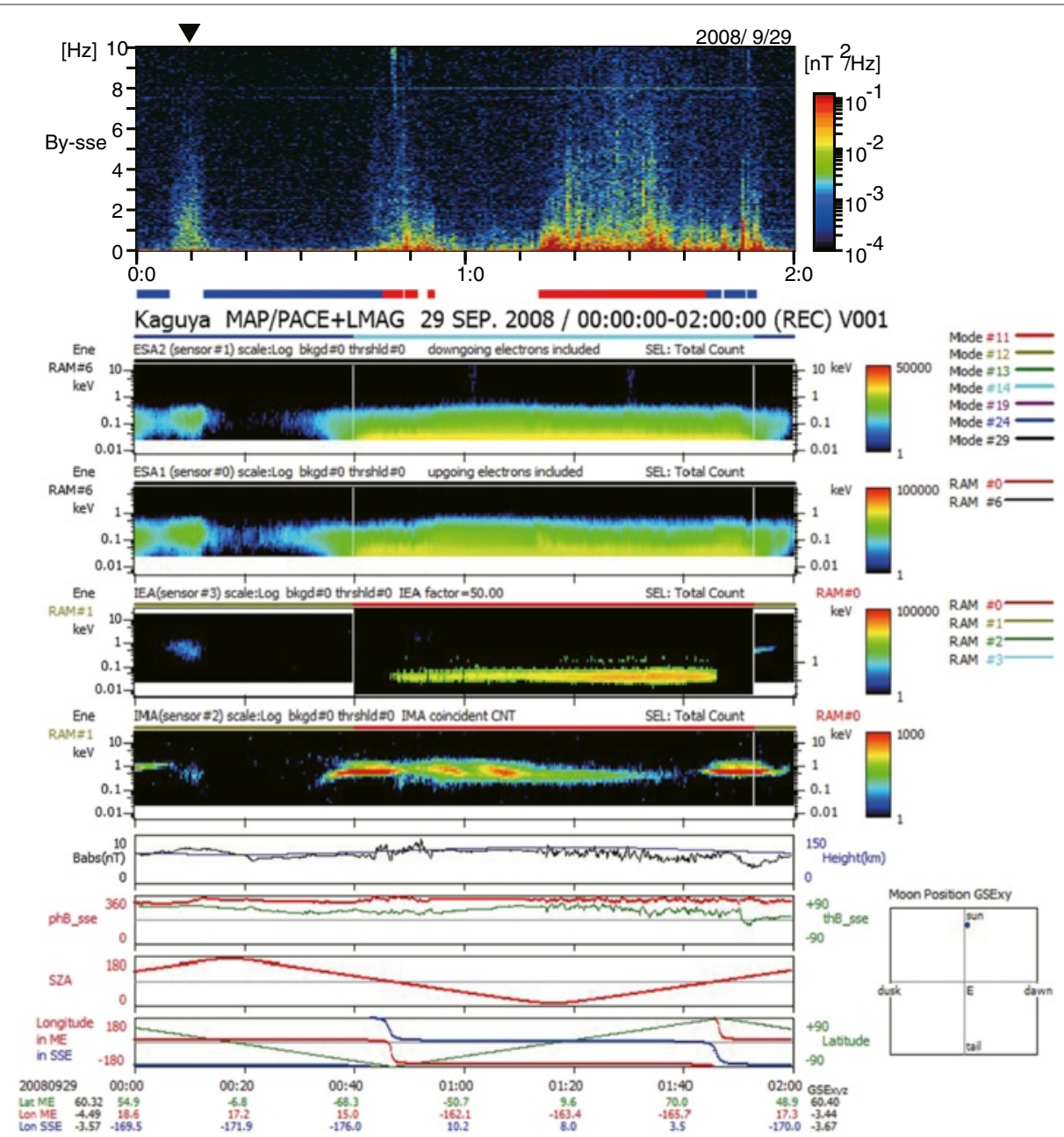

Figure 5 Dynamic spectrum of magnetic fluctuations combined with particle measurement on 29 September 2008. Top to bottom: dynamic spectrum of $y$ component $B_{y}$-sse of magnetic field in SSE frame of reference measured by MAP-LMAG magnetometer on board Kaguya, magnetic connection of Kaguya to dayside (red bars) or nightside (blue bars) surface of the moon, energy time spectra of electrons detected by ESA2 sensor (on zenith-looking panel) and ESA1 sensor (on nadir-looking panel) of MAP-PACE, energy time spectra of ions detected by IEA sensor (on zenithlooking panel) and IMA sensor (on nadir-looking panel), magnitude and directions of magnetic field measured by LMAG, solar zenith angle, and longitude of Kaguya. Wave activity was observed at 0:08 to 0:12, accompanied by type-Il protons, when spacecraft was magnetically disconnected from lunar surface. Horizontal lines at 2, 4, and $8 \mathrm{~Hz}$ in the top panel are artificial noise. 
Table 1 lists 15 events of magnetic fluctuations found in the central wake when the moon was exposed to unperturbed solar wind. These events were selected from the LMAG data set obtained in the central wake within $45^{\circ}$ of the midnight meridian and within $45^{\circ}$ of the equator in the selenocentric solar ecliptic (SSE) coordinate system, together with positive $x_{\mathrm{GSE}}$, where $x_{\mathrm{GSE}}$ is the $x$ component of Kaguya's position in geocentric solar ecliptic (GSE) coordinates. The condition $x_{\mathrm{GSE}}>$ 0 on the lunar orbit guarantees that the moon was exposed to the solar wind and was far upstream of the Earth's bow shock. The condition, $x_{\mathrm{GSE}}>0$ does not exclude the foreshock region, but most of the events in Table 1 were detected upstream of the foreshock, with only one candidate for exception $(\sharp 6)$, found downstream of the interplanetary magnetic field line tangent to the Earth's bow shock. The selection criterion of the ELF waves is a power density greater than $3 \times 10^{-3}$ $\left[\mathrm{nT}^{2} / \mathrm{Hz}\right]$ in the frequency range greater than $3 \mathrm{~Hz}$. Any abrupt changes of the magnetic field intrinsic to the solar wind, which would also produce broadband spectra, were removed from the list of candidate events by visual inspection of the waveforms obtained by the spacecraft Kaguya and ACE. ACE data obtained at 215 to $245 \mathrm{R}_{E}$ upstream of the Earth were employed to observe the waveform of the unperturbed solar wind magnetic field.

It is recognized in Table 1 that all the magnetic fluctuations were accompanied by the type-II entry solar wind protons. The average magnetic field during the events had a large $y$ component in the SSE coordinate system, which gives a favorable condition for type-II entry. The duration of the detection of the type-II protons was often longer than that of the ELF waves, suggesting that the existence of the type-II protons is not the only condition for the excitation of the waves.

\section{Control by magnetic connection}

Figure 6 shows an event that may provide a clue as to why the duration of the wave activity was shorter than that of the type-II protons. This was an event of type-II entry protons with and without magnetic connection to the lunar surface, observed by Kaguya on 4 April 2008, and reported by Nishino et al. (2013). This event is excluded from Table 1 because of the proximity to the large, abrupt changes of the interplanetary magnetic field that would cause broadband spectra. The abrupt changes are indicated by the gray dashed lines in Figure 6. The type-II protons were observed on the magnetic field lines connected to the lunar surface during the period from 18:44 to $18: 48$, as recognized from the blue bars just below the dynamic spectrum in Figure 6, then on the field lines detached from the lunar surface from 18:48 to 18:53. The wave activity was observed during the latter period, from $18: 48$ to $18: 53$, accompanied by the type-II protons on the field lines disconnected from the surface of the moon. At the same time, an enhancement of the electron energy was observed. On the other hand, the magnetic field was essentially quiet during the former period of the type-II protons on the field lines connected to the lunar surface. Although several broadband spectra are recognized

Table 1 ELF waves detected by Kaguya in deepest wake of moon

\begin{tabular}{|c|c|c|c|c|c|c|c|c|}
\hline Number & Date & Time & $\begin{array}{l}\text { Longitude } \\
\text { (SSE) }\end{array}$ & $\begin{array}{l}\text { Latitude } \\
\text { (SSE) }\end{array}$ & $\begin{array}{l}\text { Solar zenith } \\
\text { angle (deg) }\end{array}$ & $\begin{array}{l}\text { Average } B[n T] \\
\text { (in SSE) }\end{array}$ & $\begin{array}{l}\text { Type-II } \\
\text { protons }\end{array}$ & Proton detection \\
\hline 1 & $8 / 28 / 2008$ & $04: 40$ to $04: 47$ & W 141.8 & S 5.1 & 135 to 142 & $(2.2,-3.9,-0.3)$ & Yes & $04: 35$ to $04: 44$ \\
\hline 2 & $8 / 28 / 2008$ & $10: 32$ to $10: 37$ & W 142.0 & N 2.9 & 140 to 142 & $(2.8,-1.5,-0.4)$ & Yes & $10: 20$ to $10: 39$ \\
\hline 3 & $8 / 30 / 2008$ & $17: 40$ to $17: 45$ & W 143.6 & N 9.4 & 143 to 144 & $(2.4,-2.6,1.2)$ & Yes & $17: 35$ to $17: 45$ \\
\hline 4 & $8 / 30 / 2008$ & $21: 29$ to $21: 33$ & W 143.3 & N 28.9 & 133 to 139 & $(2.9,-0.7,0.9)$ & Yes & $21: 30$ to $21: 43$ \\
\hline 5 & $8 / 31 / 2008$ & $11: 22$ to $11: 25$ & W 143.9 & N 15.4 & 141 to 144 & $(3.2,-1.6,1.1)$ & Yes & $11: 13$ to $11: 25$ \\
\hline 6 & $9 / 3 / 2008$ & $22: 02$ to $22: 06$ & W 146.5 & N 34.0 & 134 to 141 & $(2.6,8.3,3.2)$ & Yes & $22: 00$ to $22: 10$ \\
\hline 7 & $9 / 4 / 2008$ & 00:01 to 00:05 & W 146.7 & N 31.7 & 135 to 142 & $(3.1,10.0,0.8)$ & Yes & $00: 00$ to $00: 10$ \\
\hline 8 & $9 / 24 / 2008$ & $06: 10$ to $06: 20$ & W 167.7 & N 3.8 & 161 to 165 & $(2.1,-5.0,-1.6)$ & Yes & $06: 10$ to $06: 18$ \\
\hline 9 & $9 / 24 / 2008$ & $10: 10$ to $10: 12$ & W 167.9 & S 8.4 & 150 to 167 & $(0.4,-6.6,-2.0)$ & Yes & $09: 57$ to $10: 17$ \\
\hline 10 & $9 / 25 / 2008$ & $21: 20$ to $21: 26$ & W 168.5 & N 36.1 & 142 to 159 & $(-2.7,6.6,-1.6)$ & Yes & $21: 17$ to $21: 26$ \\
\hline 11 & $9 / 27 / 2008$ & 08:55 to 09:00 & W 170.1 & N 4.7 & 165 to 170 & $(2.4,-4.4,-0.5)$ & Yes & 08:55 to 09:00 \\
\hline 12 & $9 / 27 / 2008$ & $12: 55$ to $13: 05$ & W 170.5 & S 7.3 & 141 to 168 & $(3.2,-5.3,-1.7)$ & Yes & $12: 55$ to $12: 58$ (EOF) \\
\hline 13 & $9 / 27 / 2008$ & $16: 42$ to $16: 47$ & W 170.0 & N 20.6 & 157 to 169 & $(1.6,-6.5,2.9)$ & Yes & $16: 42$ to $16: 47$ \\
\hline 14 & $9 / 29 / 2008$ & 00:08 to 00:12 & W 170.8 & N 28.9 & 150 to 161 & $(2.8,-3.0,5.8)$ & Yes & $00: 08$ to $00: 12$ \\
\hline 15 & 9/30/2008 & $15: 27$ to $15: 31$ & W 172.4 & N 35.8 & 144 to 155 & $(3.6,-2.0,7.8)$ & Yes & $15: 27$ to $15: 31$ \\
\hline
\end{tabular}




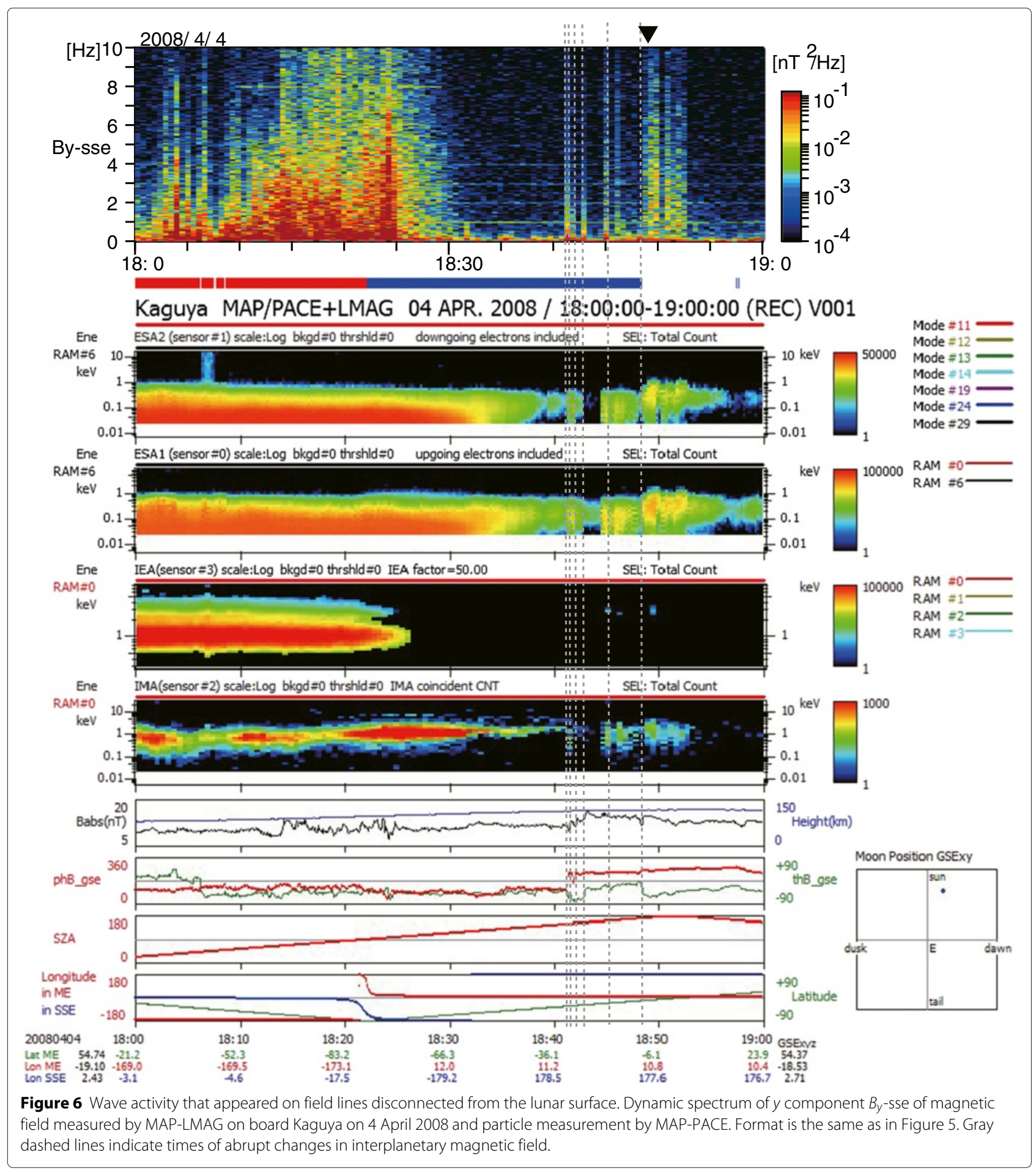

in Figure 6, for example, at 18:41, 18:43, and 18:45, they were caused by the intrinsic changes of the incident solar wind magnetic field (dashed line) and were not due to the wave activity. This suggests that the magnetic connectivity controls the electron flux and the occurrence of the ELF waves.
Table 2 lists the magnetic connection between the spacecraft and the lunar surface. Thirteen events out of 15 were observed when the spacecraft was magnetically disconnected from the lunar surface, and five of them (indicated with $\neq)$ appeared during magnetic disconnection from the lunar surface. Enhancement of the electron flux 
Table 2 Magnetic connection and enhancement of electron flux observed with ELF waves in deepest wake

\begin{tabular}{|c|c|c|c|c|c|c|}
\hline Number & Date & Time & $\begin{array}{l}\text { Protons } \\
\text { (IEA) }\end{array}$ & $\begin{array}{l}\text { Protons } \\
\text { (IMA) }\end{array}$ & $\begin{array}{l}\text { Enhancement of } \\
\text { electron flux }\end{array}$ & $\begin{array}{l}\text { Magnetic } \\
\text { connection }\end{array}$ \\
\hline 1 & $8 / 28 / 2008$ & $04: 40$ to $04: 47$ & & $1 \mathrm{keV}$ & & No \\
\hline 2 & $8 / 28 / 2008$ & $10: 32$ to $10: 37$ & $0.5 \mathrm{keV}$ & $0.5 \mathrm{keV}$ & & No $\neq$ \\
\hline 3 & 8/30/2008 & $17: 40$ to $17: 45$ & & $0.6 \mathrm{keV}$ & 0.1 to $0.3 \mathrm{keV}$ & No \\
\hline 4 & 8/30/2008 & $21: 30$ to $21: 33$ & & 0.3 to $0.7 \mathrm{keV}$ & 0.05 to $0.2 \mathrm{keV}$ & No $\neq$ \\
\hline 5 & $8 / 31 / 2008$ & $11: 22$ to $11: 25$ & & $1 \mathrm{keV}$ & 0.05 to $0.2 \mathrm{keV}$ & No $\neq$ \\
\hline 6 & $9 / 3 / 2008$ & $22: 02$ to $22: 06$ & $5 \mathrm{keV}$ & $5 \mathrm{keV}$ & $\dagger$ & Yes \\
\hline 7 & $9 / 4 / 2008$ & 00:01 to 00:05 & $3.5 \mathrm{keV}$ & $3.5 \mathrm{keV}$ & $\dagger$ & Yes \\
\hline 8 & $9 / 24 / 2008$ & 06:10 to $06: 20$ & & 0.2 to $0.6 \mathrm{keV}$ & 0.1 to $0.3 \mathrm{keV}$ & No \\
\hline 9 & $9 / 24 / 2008$ & 10:10 to $10: 12$ & & $0.5 \mathrm{keV}$ & 0.08 to $0.3 \mathrm{keV}$ & No \\
\hline 10 & $9 / 25 / 2008$ & $21: 20$ to $21: 26$ & 0.6 to $2 \mathrm{keV}$ & 0.6 to $2 \mathrm{keV}$ & $\dagger$ & No \\
\hline 11 & $9 / 27 / 2008$ & 08:55 to 09:00 & 0.3 to $0.6 \mathrm{keV}$ & & 0.1 to $0.3 \mathrm{keV}$ & No $\neq$ \\
\hline 12 & $9 / 27 / 2008$ & $12: 55$ to $13: 05$ & 0.3 to $0.5 \mathrm{keV}$ & 0.3 to $0.5 \mathrm{keV}$ & $\dagger$ & No \\
\hline 13 & $9 / 27 / 2008$ & $16: 42$ to $16: 47$ & 0.3 to $0.6 \mathrm{keV}$ & 0.2 to $1 \mathrm{keV}$ & $\dagger$ & No \\
\hline 14 & 9/29/2008 & 00:08 to $00: 12$ & $0.5 \mathrm{keV}$ & $0.5 \mathrm{keV}$ & 0.05 to $0.5 \mathrm{keV}$ & No $\neq$ \\
\hline 15 & $9 / 30 / 2008$ & $15: 27$ to $15: 31$ & & $0.5 \mathrm{keV}$ & 0.05 to $0.5 \mathrm{keV}$ & No \\
\hline
\end{tabular}

†Not clear due to intermittent observation. $¥$ Start at magnetic disconnection/end at magnetic connection.

with energy of the order of $0.1 \mathrm{keV}$ was observed in eight events out of the ten for which continuous observation of the electron spectra was available.

On the other hand, there were only two events observed on 3 and 4 September 2008, when the spacecraft was magnetically connected to the lunar surface. They were accompanied by higher energy protons of 3.5 to $5 \mathrm{keV}$. Figure 7 shows the event observed from 22:02 to 22:06 on 3 September 2008, during which the spacecraft was magnetically connected to the nightside surface of the moon, as indicated by the blue bars. The nadir angle of the magnetic field was in the range of $55^{\circ}$ to $69^{\circ}$ during the period, in contrast to other events whose nadir angles were larger than $70^{\circ}$. The energy of protons observed by the IEA and IMA was as high as $5 \mathrm{keV}$, about four to five times as large as that of the incident solar wind protons, $1 \mathrm{keV}$, calculated from the incident speed of the solar wind protons of $450 \mathrm{~km} / \mathrm{s}$ measured by ACE. This is well below the highest energy of the solar wind protons reflected by the moon after experiencing self-pickup acceleration, 8.4 times as high as that of the incident solar wind calculated for this event in the solar wind magnetic field $(2.6,8.3,3.29) \mathrm{nT}$. The search for electron enhancement was difficult because of the intermittent observations of MAP-PACE/ESA.

On the subsequent revolution of Kaguya, ELF waves were observed again on the magnetic field lines connected to the lunar surface from 0:01 to 0:05 on 4 September 2008. The energy of the type-II protons was as high as 3.5 $\mathrm{keV}$.

\section{Summary of observations}

The properties of the magnetic fluctuations are summarized as follows:

i) ELF waves in the frequency range from 0.1 to $10 \mathrm{~Hz}$ were observed by Kaguya in the deepest wake of the moon.

ii) The waves had a compressional component and showed no preferred polarization.

iii) The ELF waves were accompanied by the type-II solar wind protons that gyrate around the solar wind magnetic field with a large gyroradius, to be injected into the wake.

iv) Most of the ELF waves (13 of 15 events) were found on magnetic field lines disconnected from the lunar surface.

v) Most of the ELF waves were accompanied by injection of solar wind electrons.

vi) Two cases were detected on the field lines connected to the lunar surface, with high-energy protons.

\section{Discussion}

\section{Wave activity and magnetic anomalies}

In the previous sections, we have seen that the ELF waves were closely related to the type-II entry of the solar wind protons. As the solar wind protons are thought to be most effectively reflected by the lunar magnetic anomalies on the dayside of the moon, it is expected that the waves in the deepest wake were observed on the opposite side 


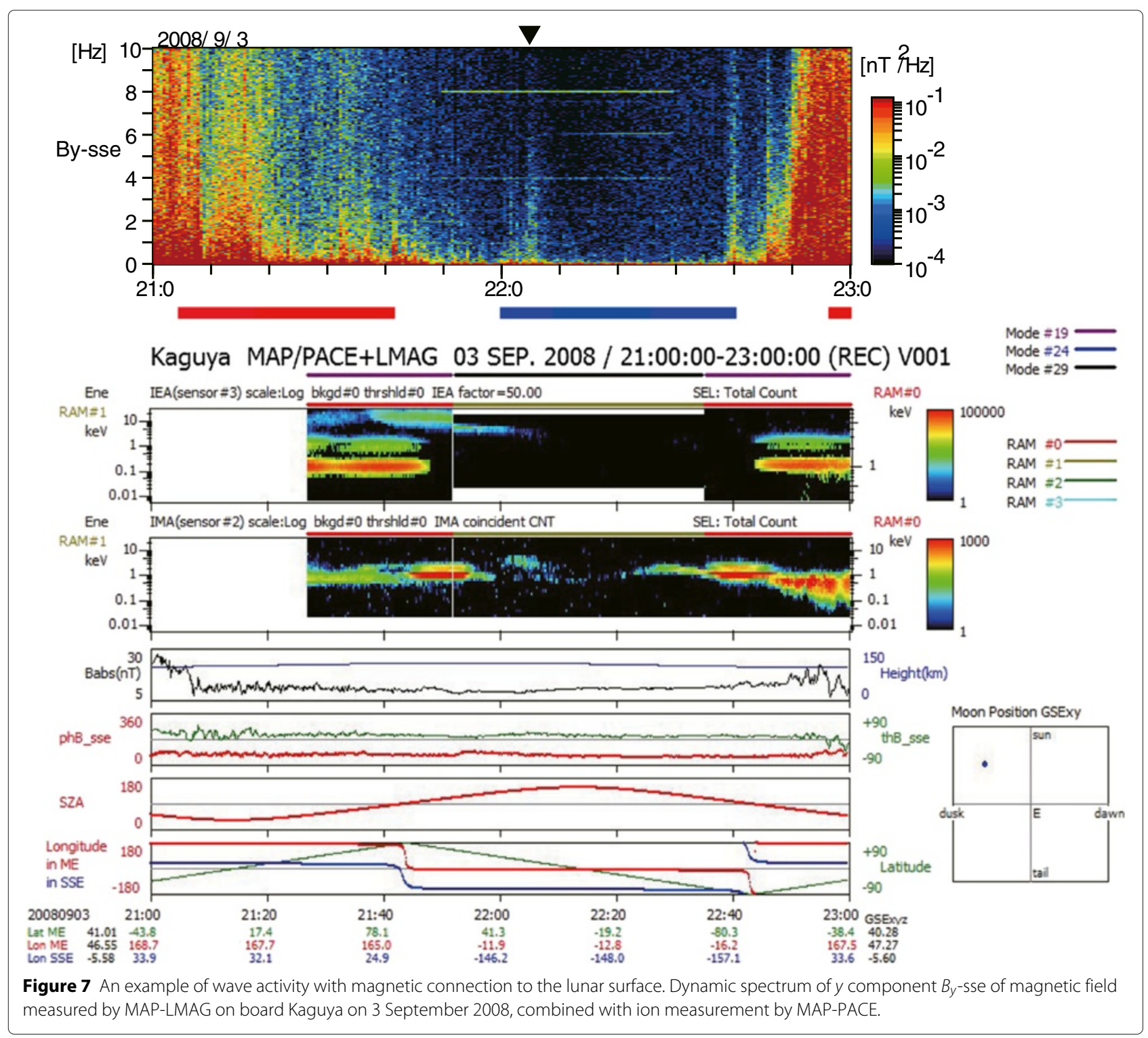

of the major magnetic anomalies. Figure $8 \mathrm{a}, \mathrm{b}$ shows the maps of the averaged power of the magnetic field fluctuations plotted in the Mean Earth/Polar Axis (ME) coordinate system, whose prime meridian ( $x$-axis) is defined by the mean Earth direction (NASA 2008). The colors indicate the power of the magnetic field fluctuations in the frequency range from 0.5 to $10 \mathrm{~Hz}$, irrespective of the selection criteria for the events in Table 1, averaged over each area of $1.5^{\circ}$ in latitude and $1.0^{\circ}$ in longitude. The wave activity detected on the dayside of the moon (Figure 8a) was enhanced above the magnetic anomalies in the southern hemisphere of the far side of the moon (Figure 8c). On the other hand, the nightside wave activity (Figure $8 \mathrm{~b}$ ), although relatively weak, was commonly observed on the near side of the moon, on the opposite side of the magnetic anomalies. This suggests that the major magnetic anomalies, when on the dayside surface, reflected the protons that were responsible for the generation of the ELF waves in the deepest wake. Figure 9, adapted from Figure 3 of Nishino et al. (2010), shows a schematic illustration of the type-II entry of protons that generate wave activity in the deepest wake.

\section{Possible generation mechanism}

The energy source of the ELF waves detected in the deepest lunar wake is thought to be the velocity difference between the type-II protons and the solar wind electrons swallowed along the magnetic field lines into the void of the wake toward the proton-rich region. It is expected that there is a significant cross-field component of velocity difference between the protons with extremely large Larmor radii and the electrons frozen-in 

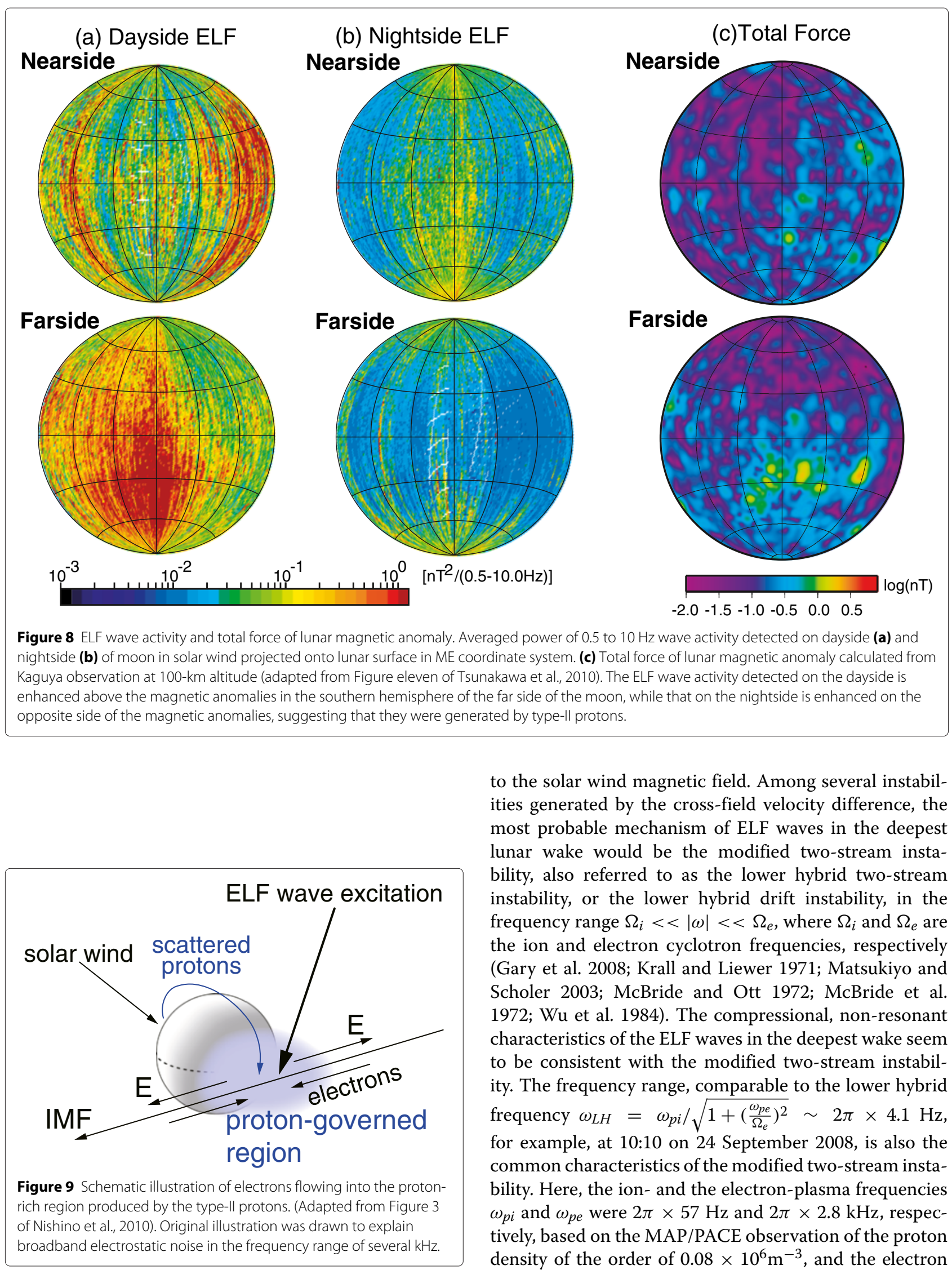

to the solar wind magnetic field. Among several instabilities generated by the cross-field velocity difference, the most probable mechanism of ELF waves in the deepest lunar wake would be the modified two-stream instability, also referred to as the lower hybrid two-stream instability, or the lower hybrid drift instability, in the frequency range $\Omega_{i}<<|\omega|<<\Omega_{e}$, where $\Omega_{i}$ and $\Omega_{e}$ are the ion and electron cyclotron frequencies, respectively (Gary et al. 2008; Krall and Liewer 1971; Matsukiyo and Scholer 2003; McBride and Ott 1972; McBride et al. 1972; Wu et al. 1984). The compressional, non-resonant characteristics of the ELF waves in the deepest wake seem to be consistent with the modified two-stream instability. The frequency range, comparable to the lower hybrid frequency $\omega_{L H}=\omega_{p i} / \sqrt{1+\left(\frac{\omega_{p e}}{\Omega_{e}}\right)^{2}} \sim 2 \pi \times 4.1 \mathrm{~Hz}$, for example, at 10:10 on 24 September 2008, is also the common characteristics of the modified two-stream instability. Here, the ion- and the electron-plasma frequencies $\omega_{p i}$ and $\omega_{p e}$ were $2 \pi \times 57 \mathrm{~Hz}$ and $2 \pi \times 2.8 \mathrm{kHz}$, respectively, based on the MAP/PACE observation of the proton density of the order of $0.08 \times 10^{6} \mathrm{~m}^{-3}$, and the electron 
cyclotron frequency $\Omega_{e}$ was $\sim 2 \pi \times 200 \mathrm{~Hz}$, according to the MAP/LMAG measurement of the magnitude of the magnetic field of $7 \mathrm{nT}$. Generation of the ELF waves by the lower hybrid drift instability might be possible because of the electron density gradient. Further work, examining the velocity difference in each event, is required in order to know whether or not the waves become unstable.

\section{Conclusion}

Magnetic fluctuations in the frequency range from 0.1 to $10 \mathrm{~Hz}$ were found in the center of the near-moon wake. They were often accompanied by solar wind protons that were once reflected by the dayside of the moon, were picked up by the solar wind electric field, then entered the deepest wake (type-II entry protons), together with the solar wind electrons injected into the proton-rich region in the wake. Since a significant cross-field velocity difference is expected between the type-II protons and the solar wind electrons, it is expected that the lower hybrid twostream instability is responsible for the generation of the waves.

\section{Competing interests}

The authors declare that they have no competing interests.

\section{Authors' contributions}

ToN, TaN, and TW performed the data analysis and manuscript preparation. HT was involved in the project planning of the magnetic field observation by Kaguya. HT and FT contributed to the analysis of the LMAG data. YS was responsible for the plasma measurements by the MAP-PACE instrument. HidS, HisS, and MM contributed to calibrating the lunar magnetometer (LMAG) on board the Kaguya spacecraft. All authors contributed to the discussion and conclusions in this study. All authors read and approved the final manuscript.

\section{Acknowledgements}

The authors are thankful to M. Nishino for developing the software to produce the E-t diagrams. We thank Kaguya MAP team for the Kaguya magnetic field and plasma observations. We also thank the ACE MAG instrument team, the ACE SWEPAM instrument team and the ACE Science Center for providing the ACE data. This work was supported by the JSPS Grant-in-Aid for Scientific Research project 24540480.

\section{Author details}

${ }^{1}$ Tohoku Institute of Technology, 35-1 Yagiyama Kasumi-cho, Taihaku-ku, 982-8577, Sendai, Japan. ${ }^{2}$ Sendai Branch Office, Tokyo Drawing Ltd., 2-1-2 Ichiban-chou, Aoba-ku, 980-0811, Sendai, Japan. ${ }^{3}$ D-CLUE Technologies Co., Ltd, IT Advancement Center, 2-5 Hibikino, Wakamatsu-ku, 808-0135, Kitakyushu, Japan. ${ }^{4}$ Department of Earth and Planetary Sciences, Tokyo Institute of Technology, 2-12-1 Ookayama, Meguro-ku, 152-8550, Tokyo, Japan. ${ }^{5}$ Department of Earth and Planetary Sciences, Faculty of Science, Kyushu University, 6-10-1 Hakozaki, Higashi-ku, 812-8581, Fukuoka, Japan. ${ }^{6}$ Department of Earth and Environmental Sciences, Kumamoto University, 391, Kurokami 2-chome, Kumamoto 860-8555, Kumamoto, Japan. ${ }^{7}$ Earthquake Research Institute, University of Tokyo, 1-1-1, Yayoi, Bunkyo-ku, 113-0032, Tokyo, Japan. ${ }^{8}$ Institute of Space and Astronautical Science, Japan Aerospace Exploration Agency, Yoshinodai 1-chome, 252-5210, Sagamihara, Japan.

Received: 4 July 2014 Accepted: 26 January 2015

Published online: 15 April 2015

\section{References}

Bosqued JM, Lormant N, Rème H, d'Uston C, Lin RP, Anderson KA, Carlson CW, Ergun RE, Larson D, McFadden J, McCarthy MP, Parks GK, Sanderson TR,
Wenzel K-P (1996) Moon-solar wind interaction: first results from the WIND/3DP experiment. Geophys Res Lett 23:1259-1262

Colburn DS, Currie RG, Mihalov JD, Sonett CP (1967) Diamagnetic solar-wind cavity discovered behind moon. Science 158:1040-1042

Gary P, Saito S, Li H (2008) Cascade of whistler turbulence: particle-in-cell simulations. Geophys Res Lett 35:02104. doi:10.1029/2007/GL032327

Krall NA, Liewer PC (1971) Low-frequency instabilities in magnetic pulses. Physical Rev A 4:2094

Lyon EF, Bridge HS, Binsak JH (1967) Explorer 35 plasma measurements in the vicinity of the moon. J Geophys Res 72:6113

Matsukiyo S, Scholer M (2003) Modified two-stream instability in the foot of high mach number quasi-perpendicular shocks. J Geophys Res 108:1459. doi:10.1029/2003JA010080

McBride JB, Ott E (1972) Theory and simulation of turbulent heating by the modified two-stream instability. Phys Lett 39A(5):363-364

McBride JB, Orr E, Boris JP, Orens JH (1972) Theory and simulation of turbulent heating by the modified two-stream instability. Phys Fluids 15:2367-2383

Nakagawa T, Takahashi Y, lizima M (2003) Geotail observation of upstream ULF waves associated with lunar wake. Earth Planets Space 55:569-580

Nakagawa T, Takahashi F, Tsunakawa H, Shibuya H, Shimizu H, Matsushima M (2011) Non-monochromatic whistler waves detected by Kaguya on the dayside surface of the moon. Earth Planets Space 63:37-46. doi:10.5047/eps.2010.01.005

Nakagawa T, Nakayama A, Takahashi F, Tsunakawa H, Shibuya H, Shimizu H, Matsushima M (2012) Large-amplitude monochromatic ULF waves detected by Kaguya at the moon. J Geophys Res 117:04101. doi:10.1029/2011JA017249

NASA GSFC (2008) A standerized lunar coordinate system for the lunar reconnaissance orbiter and lunar datesets. In: LRO Project and LGCWG White Paper. Ver 5. Goddard Space Flight Center, Greenbelt, Maryland

Ness NF, Behannon KW, Taylor HE, Whang YC (1968) Perturbations of the interplanetary magnetic field by the lunar wake. J Geophys Res 73:3421-3440

Nishino MN, Fujimoto M, Maezawa K, Saito Y, Yokota S, Asamura K, Tanaka T, Tsunakawa H, Matsushima M, Takahashi F, Terasawa T, Shibuya H, Shimizu H (2009) Solar-wind proton access deep into the near-moon wake. Geophys Res Lett 36:16103. doi:10.1029/2009GL039444

Nishino MN, Fujimoto M, Saito Y, Yokota S, Kasahara Y, Omura Y, Goto Y, Hashimoto K, Kumamoto A, Ono T, Tsunakawa H, Matsushima M, Takahashi F, Shibuya H, Shimizu H, Terasawa T (2010) Effect of the solar wind proton entry into the deepest lunar wake. Geophys Res Lett 37:12106. doi:10.1029/2010GL043948

Nishino MN, Fujimoto M, Saito Y, Tsunakawa H, Kasahara Y, Kawamura M, Matsushima M, Takahashi F, Shibuya H, Shimizu H, Goto Y, Hashimoto K, Omura Y, Kumamoto A, Ono T, Yokota S (2013) Type-Il entry of solar wind protons into the lunar wake: effects of magnetic connection to the night-side surface. Planetary Space Sci 87:106-114

Ogilvie KW, Steinberg JT, Fitzenreiter RT, Owen CJ, Lazarus AJ, Farrell WM, Torbert RB (1996) Observation of the lunar plasma wake from the WIND spacecraft on December 27, 1994. Geophys Res Lett 23:1255-1258

Owen CJ, Lepping RP, Ogilvie KW, Slavin JA, Farrell WM, Byrnes JB (1996) The lunar wake at $68 R_{L}:$ WIND magnetic field observations. Geophys Res Lett 23:1263-1266

Saito Y, Yokota S, Asamura K, Tanaka T, Akiba R, Fujimoto M, Hasegawa H, Hayakawa H, Hirahara M, Hoshino M, Machida S, Mukai T, Nagai T, Nagatsuma T, Nakamura M, Oyama K-I, Sagawa E, Sasaki S, Seki K, Terasawa T (2008a) Low-energy charged particle measurement by MAP-PACE onboard SELENE. Earth Planets Space 60:375-385

Saito Y, Yokota S, Tanaka T, Asamura K, Nishino MN, Fujimoto M, Tsunakawa H, Shibuya H, Matsushima M, Shimizu H, Takahashi F, Mukai T, Terasawa T (2008b) Solar wind proton reflection at the lunar surface: low energy ion measurement by MAP-PACE onboard SELENE (KAGUYA). Geophys Res Lett 35:24205. doi:10.1029/2008GL036077

Saito Y, Yokota S, Asamura K, Tanaka T, Nishino MN, Yamamoto T, Terakawa Y, Fujimoto M, Hasegawa H, Hayakawa H, Hirahara M, Hoshino M, Machida S, Mukai T, Nagai T, Nagatsuma T, Nakagawa T, Nakamura M, Oyama K-i, Sagawa E, Sasaki S, Seki K, Shinohara I, Terasawa T, Tsunakawa H, Shibuya H, Matsushima M, Shimizu H, Takahashi F (2010) In-flight performance and initial results of plasma energy angle and composition experiment (PACE) on SELENE (Kaguya). Space Sci Rev 154:265-303. doi:10.1007/s11214-010-9647-x 
Saito Y, Nishino MN, Fujimoto M, Yamamoto T, Yokota S, Tsunakawa H, Shibuya H, Matsushima M, Shimizu H, Takahashi F (2012) Simultaneous observation of the electron acceleration and ion deceleration over lunar magnetic anomalies. Earth Planets Space 64:83-92. doi:10.5047/eps.2011.07.011

Schubert G, Lichtenstein BR (1974) Observations of moon-plasma interactions by orbital and surface experiments. Rev Geophys Space Phys 12:592-626

Shimizu H, Takahashi F, Horii N, Matsuoka A, Matsushima M, Shibuya H, Tsunakawa H (2008) Ground calibration of the high-sensitivity SELENE lunar magnetometer LMAG. Earth Planets Space 60:353-363

Takahashi F, Shimizu H, Matsushima M, Shibuya H, Matsuoka A, Nakazawa S, lijima Y, Otake H, Tsunakawa H (2009) In-orbit calibration of the lunar magnetometer onboard SELENE (KAGUYA). Earth Planets Space 61:1269-1274

Tsugawa Y, Terada N, Katoh Y, Ono T, Tsunakawa H, Takahashi F, Shibuya H, Shimizu H, Matsushima M (2011) Statistical analysis of monochromatic whistler waves near the moon detected by Kaguya. Ann Geophys 29:889-893

Tsugawa Y, Katoh Y, Terada N, Ono T, Tsunakawa H, Takahashi F, Shibuya H, Shimizu H, Matsushima M, Saito Y, Yokota S, Nishino MN (2012) Statistical study of broadband whistler-mode waves detected by Kaguya near the moon. Geophys Res Lett 39:16101. doi:10.1029/2012GL052818

Tsunakawa H, Shibuya H, Takahashi F, Shimizu H, Matsushima M, Matsuoka A, Nakazawa S, Otake H, lijima Y (2010) Lunar magnetic field observation and initial global mapping of lunar magnetic anomalies by MAP-LMAG onboard SELENE (Kaguya). Space Sci Rev 154:219-251. doi:10.1007/s11214-010-9652-0

Wu CS, Zhou YM, Tsai ST, Guo SC, Winske D, Papadopoulos K (1984) A kinetic cross-field streaming instability. Phys Fluids 26:1259-1267

\section{Submit your manuscript to a SpringerOpen ${ }^{\circ}$ journal and benefit from:}

- Convenient online submission

- Rigorous peer review

- Immediate publication on acceptance

- Open access: articles freely available online

- High visibility within the field

- Retaining the copyright to your article

Submit your next manuscript at $\gg$ springeropen.com 